\title{
ON A CONNECTION BETWEEN KAM AND NEKHOROSHEV'S THEOREMS
}

\author{
ALESSANDRO MORBIDELLI \\ CNRS, Observatoire de la Côte d'Azur \\ BP 229, 06304 - NICE Cedex 4, France \\ ANTONIO GIORGILLI \\ Dipartimento di Matematica dell' Università di Milano \\ and Gruppo Nazionale di Fisica Matematica del CNR, \\ Via Saldini 50, 20133 Milano, Italy.
}

\begin{abstract}
Using the iteration of Nekhoroshev's theorem as a basic tool, we point out the existence of a hierarchic structure of nested domains underlying the phenomenon of diffusion. At each level we find that the diffusion speed is exponentially small with respect to the previous level. The set of KAM tori is the domain characterized by a zero diffusion speed.
\end{abstract}

\section{Introduction and statement of the result}

We consider a canonical system of differential equations with Hamiltonian

$$
H(p, q, \varepsilon)=h(p)+\varepsilon f(p, q, \varepsilon),
$$

defined on the phase space $\mathcal{D}=\mathcal{G}_{\varrho} \times \mathbf{T}^{n}$, where

$$
\mathcal{G}_{\varrho}=\bigcup_{p \in \mathcal{G}} B_{\varrho}(p)
$$

$\mathcal{G}$ being a bounded subset of $\mathbf{R}^{n}$, and $B_{\varrho}(p)$ the open ball of radius $\varrho>0$ centered at the point $p$. As usual, we denote by $p, q$ a set of action-angle variables, and by $\varepsilon$ a small parameter. The Hamiltonian is assumed to be real holomorphic in all variables.

Let $\varphi^{t}$ be the canonical flow generated by the Hamiltonian (1). A $n$-dimensional torus $\mathcal{T}$ will be said to be $(\eta, T)$-stable in case one has $\operatorname{dist}\left(\varphi^{t} P, \mathcal{T}\right)<\eta$ for all $|t|<T$ and for every $P \in \mathcal{T}$.

We state the following

Theorem: Consider the Hamiltonian (1), and assume that the unperturbed Hamiltonian $h(p)$ is convex. Then there exists $\varepsilon^{*}>0$ such that for all $\varepsilon<\varepsilon^{*}$ the following 
statement holds true: there is a sequence $\left\{\mathcal{D}^{(r)}\right\}_{r \geq 0}$ of subsets of $\mathcal{D}$, with $\mathcal{D}^{(0)}=\mathcal{D}$, and two sequences $\left\{\varepsilon_{r}\right\}_{r \geq 0}$ and $\left\{\varrho_{r}\right\}_{r \geq 1}$ of positive numbers satisfying

$$
\begin{array}{ll}
\varepsilon_{0}=\varepsilon, & \varepsilon_{r}=O\left(\exp \left(-1 / \varepsilon_{r-1}\right)\right), \\
\varrho_{0}=\varrho, & \varrho_{r}=O\left(\varepsilon_{r-1}^{1 / 4}\right),
\end{array}
$$

such that for every $r \geq 0$ one has:

(i) $\mathcal{D}^{(r+1)} \subset \mathcal{D}^{(r)}$;

(ii) $\mathcal{D}^{(r)}$ is a set of $n$-dimensional tori diffeomorphic to $\mathcal{G}_{\varrho_{r}}^{(r)} \times \mathbf{T}^{n}$, where $\mathcal{G}_{\varrho_{r}}^{(r)}$ has the form (2);

(iii) $\operatorname{Vol}\left(\mathcal{D}^{(r+1)}\right)>\left(1-O\left(\varepsilon_{r}^{a}\right)\right) \operatorname{Vol}\left(\mathcal{D}^{(r)}\right)$ for some positive $a<1$;

(iv) $\mathcal{D}^{(\infty)}=\bigcap_{r} \mathcal{D}^{(r)}$ is a set of invariant tori for the flow $\varphi^{t}$, and moreover one has $\operatorname{Vol}\left(\mathcal{D}^{(\infty)}\right)>\left(1-O\left(\varepsilon_{0}^{a}\right)\right) \operatorname{Vol}\left(\mathcal{D}^{(0)}\right)$;

(v) for every $p^{(r)} \in \mathcal{G}^{(r)}$ the torus $p^{(r)} \times \mathbf{T}^{n} \subset \mathcal{D}^{(r)}$ is $\left(\varrho_{r+1}, 1 / \varepsilon_{r+1}\right)$-stable;

(vi) for every point $p^{(r)} \in \mathcal{G}^{(r)}$ there exists an invariant torus $\mathcal{T} \subset B_{\varrho_{r}}\left(p^{(r)}\right) \times \mathbf{T}^{n}$.

Our theorem points out the existence of a direct link between the celebrated theorems of Nekhoroshev ${ }^{[4][5]}$ on the one hand, and of Kolmogorov ${ }^{[1]}$, Arnold ${ }^{[3]}$ et Moser $^{[2]}$ on the other hand. We emphasize the following three points.

A) The main technical tool of the proof is represented by the iteration of Nekhoroshev's theorem. Indeed, a careful reading of the usual proof of Nekhoroshev's theorem, and in particular of the so called geometric part, allows one to extract the following information: there exists a subset $\mathcal{D}^{(1)}$ of phase space charaterized by absence of resonances of order smaller than $O(1 / \varepsilon)$. Moreover, in this subset one can introduce new action-angle variables which give the Hamiltonian the original form (1), but with a erturbation of size $O(1 / \exp (\varepsilon))$. Nekhoroshev's theorem can be applied again to the new Hamiltonian, thus allowing one to construct a second domain $\mathcal{D}^{(2)}$. Such a procedure can be iterated infinitely many times, and this gives the sequence $\mathcal{D}^{(r)}$ of subsets of phase space, the existence of which is stated in the theorem. Nekhoroshev's stability estimates hold in every such domain, with stability time exponentially increasing at every step.

B) The iteratiove procedure converges to a set $\mathcal{D}^{(\infty)}$ of invariant tori. This set is similar to the one obtained by Arnold in his proof of KAM theorem. We are actually proving that the applicability of Nekhoroshev's theorem implies the existence of invariant tori: no further condition on the size $\varepsilon$ of the perturbation is necessary.

C) Properties (v) et (vi) imply that every $\left(\varrho^{r+1}, 1 / \varepsilon_{r+1}\right)$-stable torus is $\varrho_{r}$-close to an invariant torus. In view of the form of the sequences $\varrho_{r}$ et $\varepsilon_{r}$ given in the satatement of our theorem one has

$$
\varepsilon_{r+1}=O\left(1 / \exp \left(1 / \varepsilon_{r}\right)\right)=O\left(1 / \exp \left(\exp \left(1 / \varepsilon_{r-1}\right)\right)\right)=O\left(1 / \exp \left(\exp \left(1 / \varrho_{r}\right)\right)\right) .
$$


Thus, we actually prove that the diffusion speed is bounded by a superexponential of the inverse of the distance from an invariant torus. This is indeed in agreement with a local result that we proved in [6].

\section{Sketch of the proof}

We start with the domain $\mathcal{D}$ characterized by a constant $\varrho$, and a Hamiltonian of the form (1) with a perturbation of size $\varepsilon$; furthermore, one has two constants $M$ et $m$ such that

$$
\|A(p) v\| \leq M\|v\|, \quad|A(p) v \cdot v| \geq m\|v\|, \quad A(p)=\frac{\partial^{2} h}{\partial p_{i} \partial p_{j}}(p) .
$$

for every $v \in \mathbf{R}^{n}$. Under these conditions Nekhoroshev's theorem can be applied, which proves $(\mathrm{v})$ for $r=0$. At the same time we extract some additional information which remains usually hidden in the proof. To this end, we exploit the so called geometric part of the proof in order to prove (i), still for $r=0$. More precisely, we state that there exists a subset $\mathcal{G}_{\varrho^{\prime}}^{\prime} \subset \mathcal{G}_{\varrho}$ of the form (2), with $\varrho^{\prime}=O\left(\varepsilon^{1 / 4}\right)$, which satisfies a nonresonance condition up to a finite, $\varepsilon$-dependent order. Next, by applying Nekhoroshev's theorem, we perform a canonical transformation which introduces new action-angle variables $p^{\prime}, q^{\prime}$ on the domain $\mathcal{D}^{\prime}=\mathcal{G}_{\varrho^{\prime}}^{\prime} \times \mathbf{T}^{n}$; this proves (ii). By a standard procedure we estimate the volume of $\mathcal{G}^{\prime}$ (see [3] ), and get $\operatorname{Vol}\left(\mathcal{G}_{\varrho^{\prime}}^{\prime}\right) \geq\left(1-O\left(\varepsilon^{a}\right)\right) \operatorname{Vol}(\mathcal{G})$, where $a<1$ is a constant. The statement (iii) then follows by remarking that $\operatorname{Vol}\left(\mathcal{D}^{\prime}\right)=(2 \pi)^{n} \operatorname{Vol}\left(\mathcal{G}_{\varrho^{\prime}}^{\prime}\right)$ and recalling the volume preserving property of canonical transformations. In the new variables $p^{\prime}, q^{\prime}$ the Hamiltonian (1) takes the form

$$
H^{\prime}\left(p^{\prime}, q^{\prime}, \varepsilon^{\prime}\right)=h^{\prime}\left(p^{\prime}\right)+\varepsilon^{\prime} f^{\prime}\left(p^{\prime}, q^{\prime}, \varepsilon^{\prime}\right),
$$

with $\varepsilon^{\prime}=O(\exp (-1 / \varepsilon))$. Finally, we prove that $h^{\prime}\left(p^{\prime}\right)$ still satisfies the convexity condition with new constants $M^{\prime}=M+O\left(\varepsilon^{1 / 4}\right)$ and $m^{\prime}=m-O\left(\varepsilon^{1 / 4}\right)$.

The argument above shows that the new Hamiltonian $H^{\prime}$ satisfies the hypotheses assumed at the beginning, on a new domain $\mathcal{D}^{\prime}$. Thus, our procedure can be iterated as many times as we want. There are just a few minor consistency problems to be taken into account during the process of iteration. First of all, one usually assumes that the action domain be convex. This hypothesis must be removed, because the noresonant domain is constructed by removing the resonant region, so that it is in general non convex. A second problem is that the canonical transformation $(p, q) \rightarrow\left(p^{\prime}, q^{\prime}\right)$ introducing new action-angle variables requires a deformation and a translation of coordinates, which should be small enough in order to be consistent with the definition of the new domain. This problem is not new, since it implicitly appears already in Nekhoroshev's construction. The relevant remark here is that the deformation and the translation are of order $O\left(\varepsilon^{1 / 2}\right)$, while the radius of the balls in (ii) is bigger, 
being of order $O\left(\varepsilon^{1 / 4}\right)$. Finally, recalling that Nekhoroshev's theorem can be applied provided $\varepsilon$ is smaller than some threshold $\varepsilon^{*}$, we must prove that the trheshold for the first application of the theorem allows us to perform all the iterations, without further smallness assumptions on $\varepsilon^{*}$. This is true because the size of the perturbation decreases exponentially at every step; that is, it decreases faster than any quantity the threshold $\varepsilon^{*}$ can depend on.

Thus, by iteration of Nekhoroshev's theorem, on proves that (i), (ii), (iii) and (v) hold for every $r$. It remains to prove (iv) et (vi).

Concerning the set $\mathcal{D}^{(\infty)}$ in (iv), the proof of its existence is a straightforward adaptation of the method used by Arnold in hid proof of KAM theorem.

In order to prove (vi) we remark that for every point $p^{(r)} \in \mathcal{G}^{(r)}$, where $r$ is an arbitrary step, we can apply the procedure above starting from the domain $\tilde{\mathcal{D}}^{(r)}\left(p^{(r)}\right)=B_{\varrho_{r}}\left(p^{r}\right) \times \mathbf{T}^{n}$, all properties (i)-(v) being satisfied. This proves that there exists a subset $\tilde{\mathcal{D}}^{(\infty)} \subset \tilde{\mathcal{D}}^{(r)}\left(p^{(r)}\right)$ of invariant tori.

This concludes the sketch of the proof. A complete proof will be published elsewhere.

Acknowledgements. We thank P. Lochak for useful discussions and remarks during the preparation of the manuscript. This work has been supported by E.C. contract N. CHRX-CT93-0330/DC.

\section{References}

[1] A. N. Kolmogorov: On the preservation of conditionally periodic motions. Dokl. Akad. Nauk SSSR, 98, 527 (1954).

[2] J. Moser: On invariant curves of area-preserving mappings of an annulus, Nachr. Akad. Wiss. Göttingen Math. Phys. Kl. 2, 1 (1962).

[3] V. I. Arnold: Proof of a theorem of A. N. Kolmogorov on the invariance of quasi-periodic motions under small perturbations of the Hamiltonian. Russ. Math. Surv., 18, 9 (1963).

[4] N. N. Nekhoroshev: Exponential estimates of the stability time of nearintegrable Hamiltonian systems, Russ. Math. Surveys, 32, 1 (1977).

[5] N. N. Nekhoroshev: Exponential estimates of the stability time of nearintegrable Hamiltonian systems, 2. Trudy Sem. Petrovs., 5, 5 (1979).

[6] A. Morbidelli and A. Giorgilli: Superexponential stability of KAM tori, J. Stat. Phys., in press. 